\title{
ÉDITORIAL
}

\section{Natures, Sciences, Sociétés : premier dossier}

\author{
MARCEL JOLIVET, JEAN-MARIE LEGAY, GÉrARD MÉGIE
}

F ait nouveau, le sommaire de ce numéro de Natures, Sciences, Sociétés fait état d'un "dossier" (sciences sociales et médecine : à propos des malades du sida). Cette innovation répond au désir de pouvoir centrer l'attention sur un thème ou une question sans tomber dans les difficultés et les rigidités de numéros spéciaux. D'autres dossiers suivront.

Celui de ce numéro porte sur certaines dimensions sociales de la maladie et, par voie de conséquence, sur une confrontation entre les sciences sociales et la médecine. D'où son intitulé. Le sida est le cas de figure considéré. Ce dossier paraît au moment même où a lieu, à Vancouver la $11^{\mathrm{e}}$ Conférence Internationale sur le sida.

L'infection par le VIH mérite une attention particulière en raison de son actualité et de sa gravité bien sûr, mais aussi en ce qu'elle représente un exemple particulièrement révélateur des rapports entre sciences biologiques et société par le biais de la maladie. C'est la raison pour laquelle on l'a retenue ici. La soudaineté avec laquelle elle s'est révélée dans un contexte sociétal où prévalait l'idée que la médecine mettait désormais I'humanité à l'abri des maladies mortelles, et I'ampleur qu'elle a prise, la mettant rapidement au rang des grandes pandémies qu'a connues l'humanité, ont brisé un des mythes des sociétés modernes et provoqué un choc collectif en rappelant que, pas plus que I'histoire sociale, politique..., I'histoire naturelle de l'homme n'était terminée : le sida apparaît comme la peste ou le choléra de nos temps modernes. Le fait que les sciences biologiques et médicales aient, au début, été prises au dépourvu par manque de connaissances et que les moyens préconisés, tant pour se prémunir contre la maladie que pour en guérir, se soient avérés inopérants, a agi dans le même sens en mettant dramatiquement en évidence les limites actuelles d'une science censée ne plus en avoir. Le caractère très particulier des formes jugées originelles de la contamination, qu'il s'agisse d'une pratique médicale (la transfusion sanguine) ou de comportements sociaux "à risques" (I'homosexualité et la toxicomanie), puis la généralisation du risque à l'ensemble de la population ont immédiatement fait du sida une question de société, voire une question politique; et ceci d'autant plus que des mouvements organisés (comme les associations d'hémophiles et le mouvement gay) et l'État se sont trouvés impliqués.

Durant la première décennie de son histoire, le sida a donné lieu à toute une dynamique sociale dans laquelle se sont mêlés une évolution extrêmement rapide des connaissances biologiques concernant la maladie (comme la découverte de l'activité du virus dans la phase asymptomatique de la séropositivité), des progrès considérables dans le traitement des maladies dites "opportunistes" qui résultent de la déficience immunitaire, puis de l'immuno-déficience elle-même, mais aussi de constants débats sur les politiques de prévention et de soins à suivre, sur les principes qui doivent les guider et les moyens qu'il convient de leur consacrer. Le dossier du sang contaminé et les procès en série auxquels il a donné lieu font évidemment partie de cette chronique. Tout ceci est à l'arrière-plan de ce dossier.
Dans l'éditorial du numéro 4, 1994 de Natures, Sciences, Sociétés, Jean-Paul Moatti attirait l'attention sur les risques d'une stigmatisation croissante des personnes séropositives, qui était en germe dans les conclusions de la précédente grande conférence internationale consacrée au sida (Yokohama, août 1994). Les conclusions d'une récente enquête semblent aller dans le même sens.

Dans le dossier présenté dans ce numéro de Natures, Sciences, Sociétés, il a paru opportun et intéressant d'opposer à ce regard externe, facilement excluant, sur la maladie, le vécu même de leur maladie par les personnes séropositives. Comment les personnes atteintes peuvent-elles prendre en charge leur état, face à cette maladie chronique très particulière débouchant à terme sur la mort, mais incertaine quant à la durée de ce terme, comportant une phase asymptomatique qui pousse le malade à rejeter l'idée même de la maladie, sujette à une évolution rapide des connaissances qui modifie en permanence la représentation biologique et immédiate que l'on s'en fait et qui accroit les incertitudes dans le même temps où elle semble offrir des voies de salut, stigmatisante en raison de sa gravité, des images auxquelles elle est associée et de la crainte de la contagion qu'elle suscite? L'idée ici est, aux côtés des points de vue de l'opinion et des médecins, de donner corps à ceux des malades eux-mêmes qui sont généralement - et paradoxalement - les grands oubliés de l'affaire. L'intérêt de cet apport des sciences sociales est d'éclairer d'un jour nouveau la pratique médicale ellemême. 